\title{
Environmental Data-Driven Performance-Based Topological Optimisation for Morphology Evolution of Artificial Taihu Stone
}

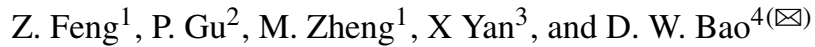 \\ ${ }^{1}$ Suzhou University of Science and Technology, Jiangsu 215000, China \\ 2 Architectural Association, London WC1B 3ES, UK \\ 3 Centre for Architecture Research and Design, University of Chinese Academy of Science, \\ Beijing 100190, China \\ 4 School of Architecture and Urban Design, Centre for Innovative Structures and Materials, \\ RMIT University, RMIT University, Melbourne 3000, Australia \\ nic.bao@rmit.edu.au
}

\begin{abstract}
Taihu stone is the most famous one among the top four stones in China. It is formed by the water's erosion in Taihu Lake for hundreds or even thousands of years. It has become a common ornamental stone in classical Chinese gardens because of its porous and intricate forms. At the same time, it has become a cultural symbol through thousands of years of history in China; later, people researched its spatial aesthetics; there are also some studies on its structural properties. For example, it has been found that the opening of Taihu stone caves has a steady-state effect which people develop its value in the theory of Poros City, Porosity in Architecture and some cultural symbols based on the original ornamental value of Taihu stone. This paper introduces a hybrid generative design method that integrates the Computational Fluid Dynamics (CFD) and Bi-directional Evolutionary Structural Optimization (BESO) techniques. Computational Fluid Dynamics (CFD) simulation enables architects and engineers to predict and optimise the performance of buildings and environment in the early stage of the design and topology optimisation techniques BESO has been widely used in structural design to evolve a structure from the full design domain towards an optimum by gradually removing inefficient material and adding materials simultaneously. This research aims to design the artificial Taihu stone based on the environmental data-driven performance feedback using the topological optimisation method. As traditional and historical ornament craftwork in China, the new artificial Taihu stone stimulates thinking about the new value and unique significance of the cultural symbol of Taihu stone in modern society. It proposes possibilities and reflections on exploring the related fields of Porosity in Architecture and Poros City from the perspective of structure.
\end{abstract}

Keywords: Bi-directional Evolutionary Structural Optimization (BESO) . Intricate Architectural Form - Computational Fluid Dynamics (CFD) · Poros City $\cdot$ Porosity 


\section{Introduction}

Taihu stone (Fig. 1) [3] is limestone and the water's erosion in Taihu Lake for hundreds or even thousands of years makes it porous and intricate. It has become a common ornamental stone in classical Chinese gardens and a symbol of Chinese culture for hundreds of years [1]. This research posits an innovative design methodology, including computational fluid dynamics (CFD) and bi-directional evolutionary structural optimisation (BESO), to design new artificial Taihu stones. The focus of this paper is the experiment to design a new Taihu stone with different parameters. This research contributes to Porosity Crafts and The Theory of Porous City [2], Porosity in Architecture from a structural standpoint and cultural symbols of Taihu stone in society.

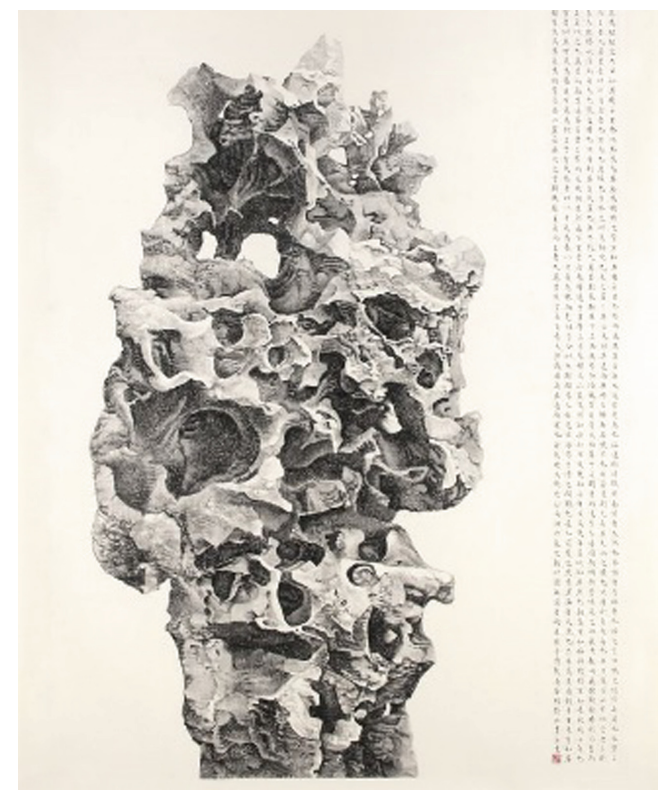

Fig. 1. Taihu stone painting

\section{Design Methodology}

\subsection{Principle of Experiment}

The experiment of "Taihu stone" reveals a cross-iteration process of CFD simulation [4-6] and topology optimisation (BESO) [7, 8]. The whole process consists of three parts: $a$. Test of Taihu Lake's original fluid condition; $b$. Pressure analysis of original mesh by CFD simulation; c. Topology optimisation based on pressure analysis. The fluid condition will change each time after the change by topology optimisation (Fig. 2); thus, iteration happens from $a$ to $c$ again (the flow chart is seen in Fig. 3). 

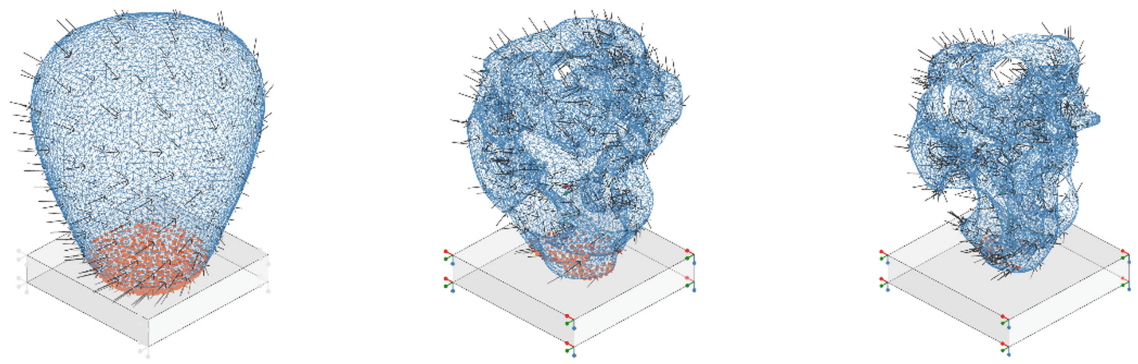

Fig. 2. Boundary Conditions during the process of topological optimisation

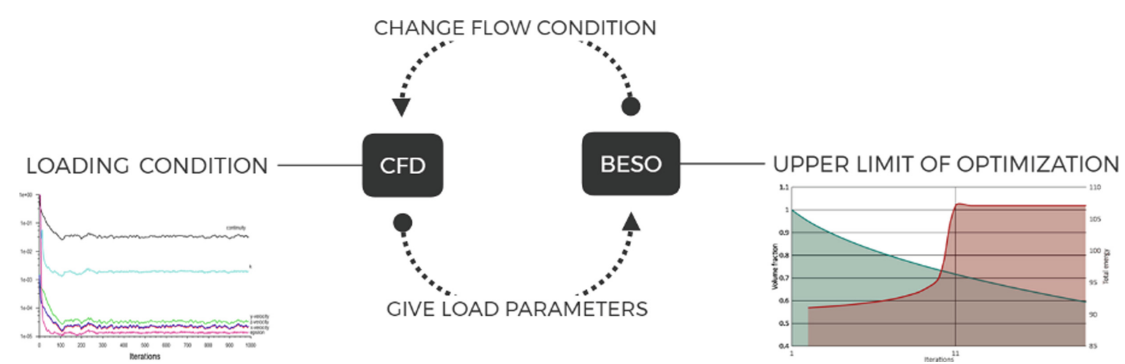

Fig. 3. Flow chart of CFD simulation and topology optimisation

\subsection{Environment Parameters of Experiments}

Studies [9-11] indicate the average depth of Taihu Lake, which is a dead lake with no current, is $1.8 \mathrm{~m}$, and the flow direction and velocity are mainly related to the speed of the prevailing wind over Taihu Lake. These studies have shown that, at the bottom of Taihu Lake, where Taihu stone is, the direction of wind-induced current is over $90 \%$ likely to be opposite to the wind above Taihu Lake, and the velocity of lower flow is relatively slower than the wind above.

Therefore, the CFD simulation of water flow in this paper applies data of the perennial water flow direction and velocity at the bottom of Taihu Lake, identified by Suzhou and Shanghai's prevailing wind direction and velocity (called SuHu area) in China.

Among the widely accepted views on the formation of the Chinese Taihu stone [1], water acidity is important for the erosion of limestone such as Taihu stone. The part that is corroded by acid is mainly dense calcium carbonate and easy to wash away by water currents. Moreover, this part is a variable that is difficult to determine its proportion and location in BESO. This paper calculates the part directly corroded by the acid in the water and eliminated by the water flow as a random ratio parameter. 


\subsection{Special Parameters Tested in the Experiments}

\subsubsection{Percentage of Low-Density Volume Compared with the Whole Volume in Taihu Stone}

It should be noted that each part of the stone's density will also affect the experi-ment's influence in reality [1]. Thus, a new constant is set to reveal the phenome-non called 'percentage of low-density part'. As a result, a fixed constant percentage of mesh will undoubtedly be eliminated in every turn of iteration, closer to the ac-tual result.

\subsubsection{Percentage of Elimination Volume Compared with the Whole Volume During the Process of Topological Optimisation}

In nature, both stone and water changes should happen simultaneously, so the interval time between each step of iteration should be extremely short, which is unrealistic in software operation currently. Thus, we will try different volume reduction to reveal the result of a certain interval before the next iteration in which fluid condition refresh. The experiment catalogue will be analysed in the next chapter.

\subsubsection{Minimum Radius of Influence Number (Rmin)}

Rmin is a parameter during BESO [12] that will affect the outcome of the calculation. Refer to new research of topology optimisation, and the result will be different from different settings, including Rmin, which means there is over one solution.

\subsection{Self-criticism of the Experiment Methodology}

The whole simulation can be regarded as a certain ideal condition of the formation of Taihu stone. The core factor should be noted that the principle of BESO is not entirely in compliance with the reality of volume reduction of Taihu stone because the change of stone in the river contains both structure optimisation and some erosion/corrosion process. However, in a sense, the experiment here proposed should be more conducive to structural stability.

\section{Quantitative Definition of Physical Characteristics of Artificial Taihu Stone with the Parametric Method}

\subsection{Criteria of Traditional Aesthetics of Chinese Taihu Stone}

According to the literature $[13,14]$ on the cultural connotation of the artistic symbol of "Taihu stone", the ancient Chinese created a set of theoretical principles and proposed the standard of phase stone. The four elements of "Shou, Lou, Zhou, Tou" were used to judge the value of Taihu stone.

"Shou" refers to the ingenious structure of Taihu stone, supporting the shape with the least amount of material, which is similar to BESO's effect [15-17]. As the number of iterations increases, the volume decreases, but the structure will always be one of the optimal solutions; "Lou" means that most of the holes in the Taihu stone are connected, 
which is a flowing space. "Zhou" is a judgment of formal aesthetics, which mainly refers to the undulating rhythm of the shape of Taihu stone - pattern in the strange and the rhyme in the difference, which parameters cannot quantify. We adopted the design of the initial prototype of Taihu Stone to reach this standard as much as possible and expressed it in the form of a model in this research. The object of "Tou" evaluation is the material characteristics of Taihu stone, which cannot be achieved by the CFD \& BESO method, nor is it the focus of this experiment, but the later construction materials can reflect it.

In conclusion, two aesthetic factors of "Zhou" (i.e., "wrinkling textures and furrows") and "Tou" (i.e., "passing through or transparency") need to be expressed by images, while the remaining two spatial factors of "Shou" (i.e., "leanness or thickness") and "Lou" (i.e., "eyes or hollowness") are expressed in a parametric way.

\subsection{Parametric Definition of "Shou"}

In this paper, the number of spatial nodes is used to test the complexity of the spatial topology, which is "Shou", higher values indicates that the spatial topology is more complex and the ingenuity of the structure.

\subsection{Parametric Definition of "Lou"}

"Lou", explained by the original Reference, is the interconnection between the holes in the Taihu stone. Combined with the actual survey of Taihu stone, we found that it is unadvisable to use the connectivity rate of holes $=n / N$ (N represents the total number of holes, and $\mathrm{n}$ represents the number of interconnections) to prove "Lou" because the actual Taihu stone is not that the more holes connected to the middle, the higher the value "Lou" of the Taihu stone, which is also related to the size of the connecting space of the Taihu stone. The extent of hollowness relates to not only the ratio of interconnections but also the size of connected holes.

Referring to the cell division method in biology, the ratio of volume to surface area is preferable to show the Taihu stone's connectivity. For example, when an organism grows, it is because its cells are dividing not getting bigger, it is challenging to keep up with taking in the extra nutrients it needs and expelling more waste, which means as the cell gets bigger, it has less surface area compared to its size - the surface area to volume ratio of the cell decreases. Cell division solves the problem of increasing size by reducing cytoplasm volume in the two daughter cells and dividing up the duplicated DNA and organelles, thereby increasing the surface to volume ratio of the cells. In this case, treating cells as a whole like original Taihu stone, then the division can be regarded as the connectivity of holes in Taihu stone, which means more cell divisions lead to higher connectivity rate of holes, mathematically, increasing surface to volume ratio leads to higher connectivity rate of holes. From this analysis method, if the cell's growth rate is stable, the higher the difference between surface and volume, the higher the cell division rate (indicates, the higher connectivity rate of holes in Taihu stone).

This research comprehensively selects the optimal solution of Taihu stone according to respective weights of 50\% and combined with the visual images from the two parameters above. 


\begin{tabular}{|c|c|c|c|c|c|c|c|}
\hline & $\begin{array}{l}\mathrm{Vf}=90 \% \\
\text { Rnd }=50 \% \\
\text { Rmin }=3 \times \text { Size }\end{array}$ & $\begin{array}{l}\mathrm{Vf}=80 \% \\
\text { Rnd }=50 \% \\
\text { Rmin }=3 \times \text { Size }\end{array}$ & \begin{tabular}{|l|}
$\mathrm{Vf}=70 \%$ \\
$\mathrm{Rnd}=50 \%$ \\
$\mathrm{Rmin}=3 \times$ Size \\
\end{tabular} & $\begin{array}{l}\mathrm{Vf}=80 \% \\
\text { Rnd }=40 \% \\
\text { Rmin }=3 \times \text { Size }\end{array}$ & \begin{tabular}{l|}
$\mathrm{V} f=80 \%$ \\
$\mathrm{Rnd}=30 \%$ \\
Rmin $=3 \times$ Size
\end{tabular} & \begin{tabular}{l|}
$\mathrm{V} f=80 \%$ \\
$\mathrm{Rnd}=40 \%$ \\
$\mathrm{Rmin}=2 \times$ Size \\
\end{tabular} & $\begin{array}{l}\mathrm{Vf}=80 \% \\
\mathrm{Rnd}=40 \% \\
\mathrm{Rmin}=1 \times \text { Size }\end{array}$ \\
\hline \multicolumn{8}{|l|}{ Iteration1 } \\
\hline & Test_1_1 & Tost_2_1 & Teot_3_1 & Test_4_-1 & Test__5_1 & Toet_-6_1 & Test__?_1 \\
\hline \multicolumn{8}{|l|}{ Iteration2 } \\
\hline & Test_1_2 & Test_z__2 & Test_3__2 & Test_4__2 & Test_5_2 & Teet_6__2 & Teet_.7_2 \\
\hline \multicolumn{8}{|l|}{ Iteration 3} \\
\hline & Test_1__3 & Test_2_.3 & Test_3_-3 & Test_4__3 & Test_5_-3 & Test_6_-3 & Trest_t_-3 \\
\hline \multicolumn{8}{|l|}{ Iteration4 } \\
\hline & Test_1_-4 & Toet___4 & Teet__3_4 & Test_4_4 & Teet_5_4 & Teet_6_-4 & Test.7-34 \\
\hline \multicolumn{8}{|l|}{ Iteration5 } \\
\hline & Test__1_5 & Test_2.5 & Test_3_5 & Test_4_-5 & Test_5_5 & Teet_6_-5 & Test.7.5 \\
\hline \multicolumn{8}{|l|}{ Iteration6 } \\
\hline & Test_1_6 & Test___6 & Test_3_6 & Teot__4_6 & Teot_5_6 & Teet_6__6 & Teot_t_-6 \\
\hline \multicolumn{8}{|l|}{ Iteration7 } \\
\hline & Test_1_-7 & Test_2.7 & Test_3_7 & Test_4_-7 & Test_5_t & Test_6_t & Test_7_t \\
\hline \multicolumn{8}{|l|}{ Iteration8 } \\
\hline & Tost_1_.8 & Test____8 & Test_3_8 & Test_4_8 & Tost_5_.8 & Tost_6__8 & Tost____8 \\
\hline \multicolumn{8}{|l|}{ Iteration9 } \\
\hline & Test_1_9 & Test_2_.9 & Tost_3_-9 & Test_4_9 & Tost_5_9 & Tost_6.9 & Tost_?_9 \\
\hline Iteration 10 & S9 & cos & 83 & & औद & \%कs & \\
\hline & Test_1_10 & Teot_2.18 & ect.3.18 & Teot_4_18 & & Tect_6_10 & 10 \\
\hline
\end{tabular}

Fig. 4. The profile results of 10 iterations of stones with different parameters of "Lou" 


\section{Screening and Evaluation of Artificial Taihu Stone Through Experiment}

\subsection{The Degree of Complexity of the Spatial Topology}

The experience uses the same piece of Taihu stone to experiment with controlling variables for the above-mentioned main parameters. (Estimate the theoretical range of the parameter range before the experiment). For seven different data, 70 different results are obtained after ten iterations of each, and their cross-sectional forms are recorded as shown in Fig. 4. Furthermore, take the parameter of Volume Fraction (Vf) $=80_{-}$ Random ratio $($ Rnd $)=40 \%$ _ Minimum Radius of Influence Number $($ Rmin $)=1 \times$ Size to illustrate the iterative calculation process of the experiment (seen in Fig. 5).

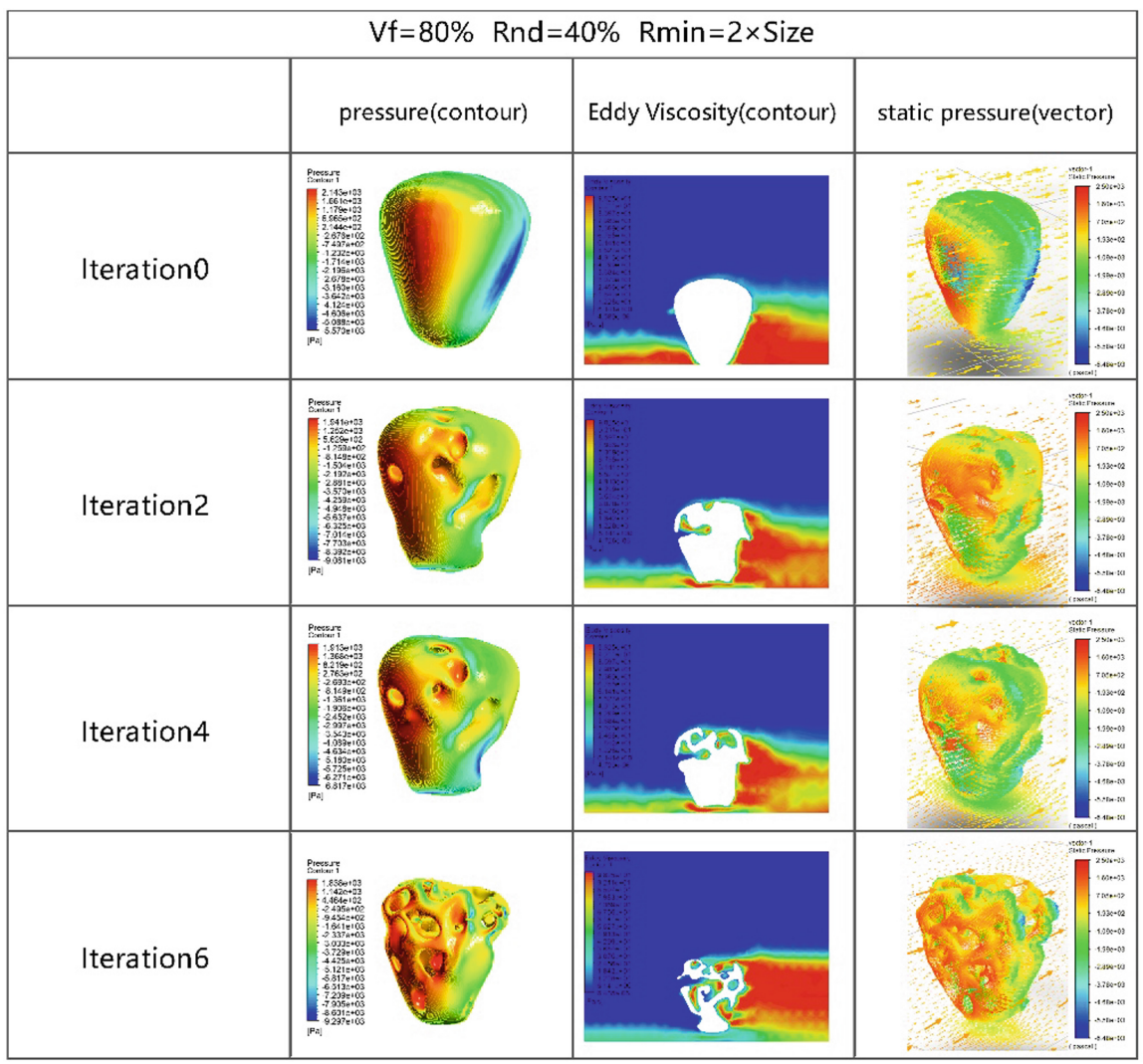

Fig. 5. Iterative calculation process in $\mathrm{CFD}$

In this paper, spatial nodes are the joints in the artificial Taihu stone skeleton to show the degree of complexity in Taihu stone (Fig. 6).

To find the number of spatial nodes of each Taihu stone. Moreover, draw a line chart (seen in Fig. 7). 

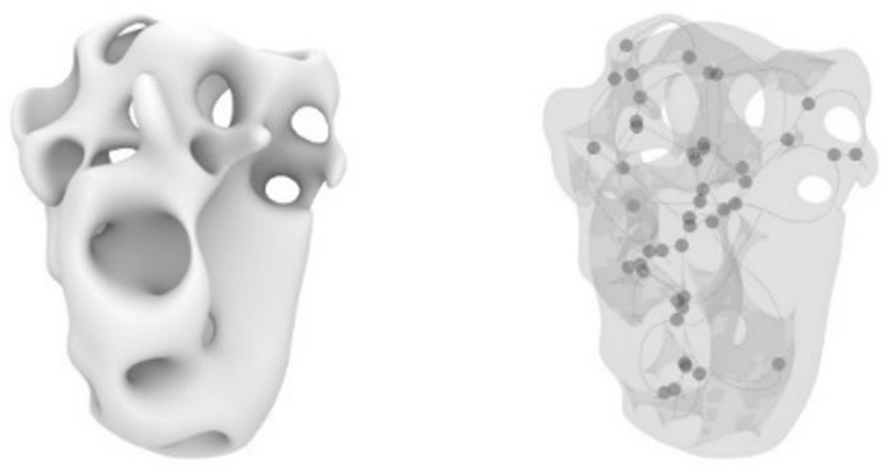

Fig. 6. Spatial nodes in artificial Taihu stone
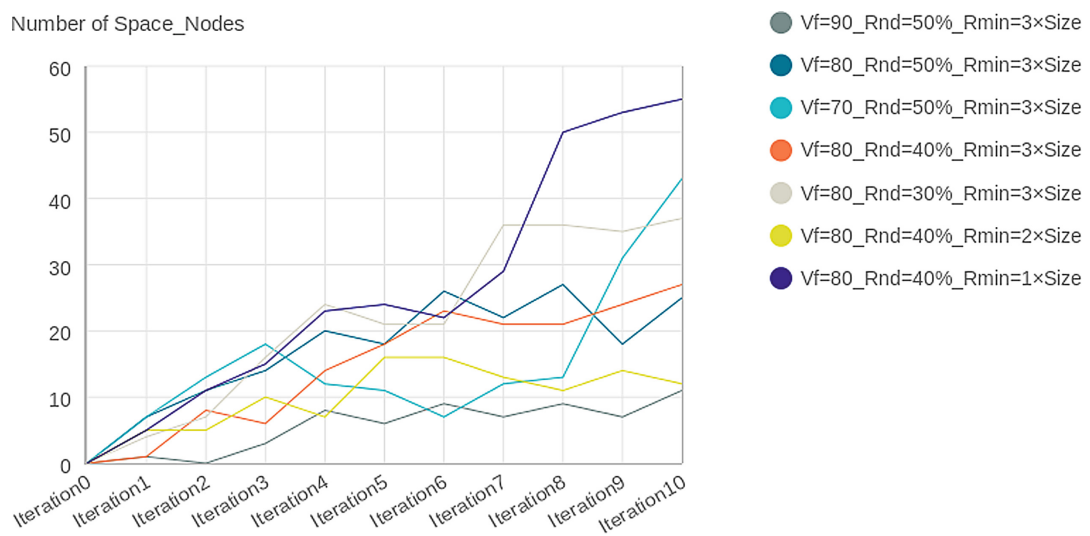

Fig. 7. Numbers of spatial nodes of each Taihu stone

According to the line chart:

1. Vf $=80 \_$Rnd $=40 \% \_$Rmin $=1 \times$ Size has the highest value of average spatial nodes during ten times iterations, and Vf $=90 \_$Rnd $=50 \% \_$Rmin $=3 \times$ Size has the least average number of spatial nodes during ten times iterations.

2. When the values of Rmin and Rnd are invariant, the higher the value of $\mathrm{Vf}$, the more complicated the final generated space, while the data of $\mathrm{Vf}=70$ is unstable.

3. When the values of Rmin and Vf are invariant, the smaller the value of Rnd, the more complicated the generated space.

4. When the values of Vf and Rnd are invariant, the space complexity: $2 \times$ size $<3 \times$ size $<1 \times$ size. (size: element size)

\subsection{The Degree of Connectivity Between the Holes}

In this paper, the ratio of the difference between the standard value and the experiment value to the standard value is used to express the degree of connectivity between holes. 
Compare the area to volume ratio of the experiment with the area to volume ratio when the $\mathrm{Vf}=90 \%, \mathrm{Vf}=70 \%, \mathrm{Vf}=80 \%$, data shown in charts. To make the data more intuitive, division level is used to show the connectivity between the holes (seen in Fig. 8).

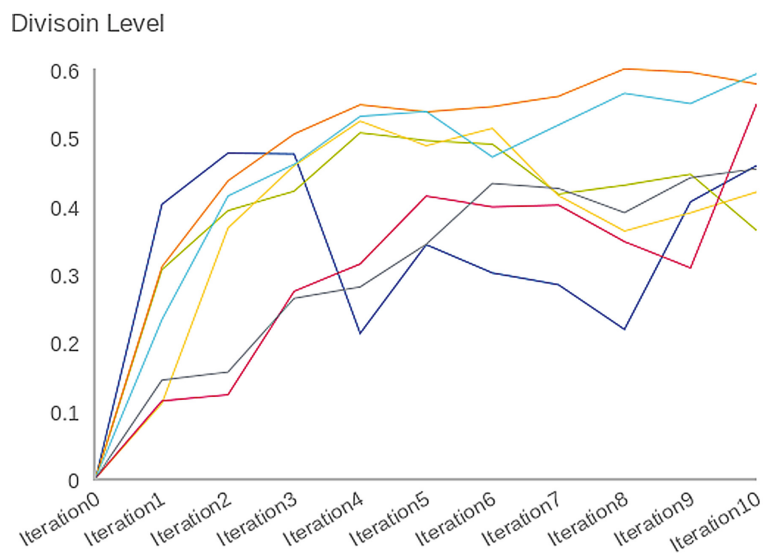

Vf $=70 \_R n d=50 \% \_R m i n=3 \times$ Size Vf $=80 \_$Rnd $=50 \%$ Rmin $=3 \times$ Size V $f=80 \_$Rnd $=40 \% \_$Rmin $=3 \times$ Size $V f=80 \_R n d=30 \% \_R m i n=3 \times$ Size $V f=80 \_R n d=40 \% \_R m i n=2 \times$ Size $V f=80 \_R n d=40 \% \_R \min =1 \times$ Size $V f=90 \_R n d=50 \% \_R m i n=3 \times$ Size

Fig. 8. Division level of a different situation

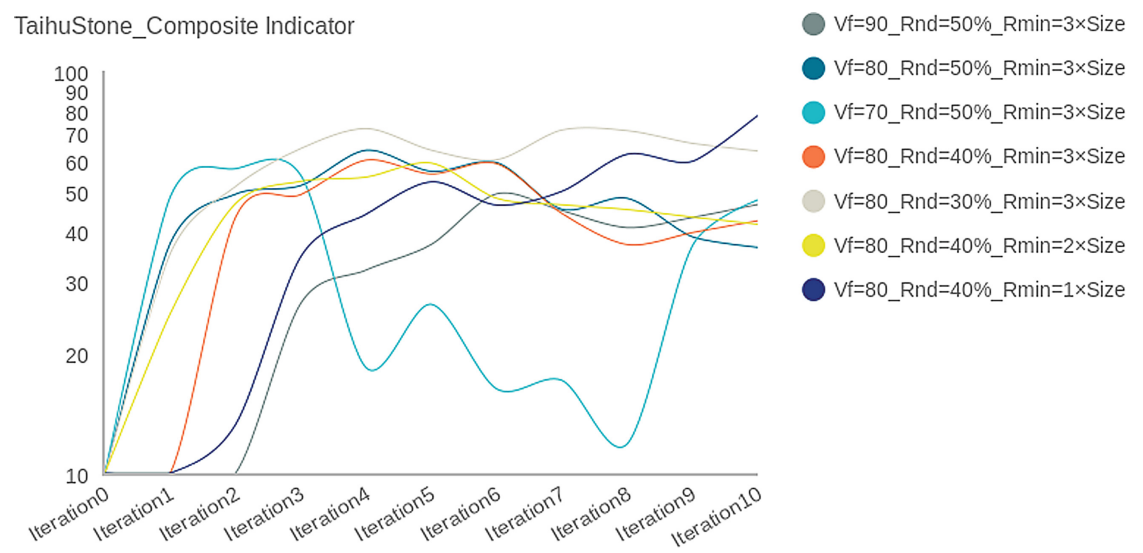

Fig. 9. A composite indicator of Taihu stone

To conclude the charts above (Fig. 9):

1. All data will first reach its peak in the iterative process, then decline till eventually stabilised. Vf $=80 \_$Rnd $=40 \% \_$Rmin $=3 \times$ Size has the highest peak and the highest average connectivity rate when stabilised.

2. $\mathrm{Vf}=70 \_\mathrm{Rnd}=50 \% \_\mathrm{Rmin}=3 \times$ Size has the earliest peak, and the value of the average connectivity rate when stabilised is the lowest.

3. $\mathrm{Vf}=80 \_$Rnd $=40 \% \_$Rmin $=1 \times$ Size has a minimum value of peak. 
4. When the values of Rnd and Rmin are invariant, the smaller the Vf, the earlier the peak appears and the higher the peak value.

5. When the values of Vf and Rmin are invariant, though Rnd does not affect the time when the peak appears, the smaller the value of Rnd, the higher the value of the peak.

6. When the Vf and Rnd values are invariant, the peak value shows the extent of connectivity between holes in Taihu stone and the average value when stabilised: 2 $\times$ size $<3 \times$ size $<1 \times$ size.

\section{The Value of Artificial Taihu Stone in Fields of Crafts and Porous Space}

\subsection{Porosity Crafts}

In this article, relative design is made by new material like stainless steel and inlaid drawn steel wire or glass fibre, new technology like sound, light, electricity to extract the beauty of shape from Taihu stone. Using parametric design methods through CFD\&BESO, the Taihu stone structure gets improved and can provide more possibilities (seen in Fig. 10). The definition as a symbol of aesthetics was then researched in the following parts to discuss how traditional crafts can have a new way of living with contemporary society.
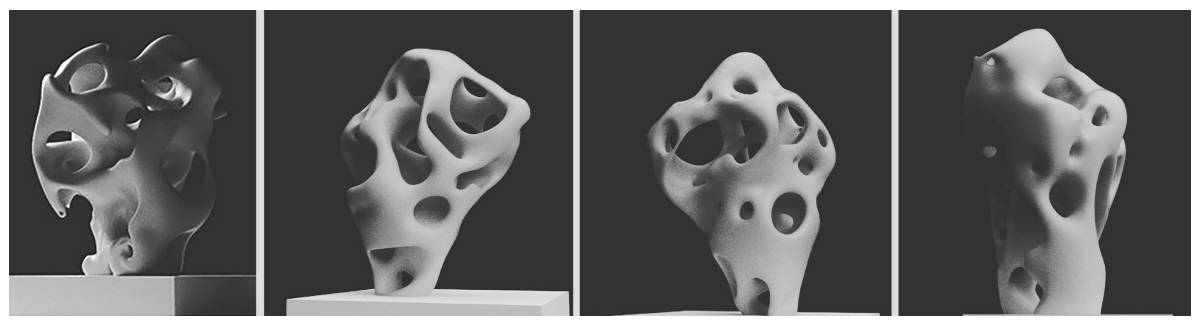

Fig. 10. Parametric Taihu stone samples

\subsection{Porosity Architecture}

Porosity in Taihu stone, featured in porous shape and façade, can be used in modern architecture design [18]; the sample of porosity architecture. In Suzhou traditional gardens, Taihu stone has an architectural contribution in light changes and circulation connection; different interpretation methods can be absorbed and improved in architecture design.

\section{a) Porosity City}

In today's city organisation, as the addition of single building units, Poros City [2] tries to get a holistic result with uniform density. We try to conclude a series of space prototype and typical ways of combination in Taihu stone. As Fig. 11 shows, the comparison was made between diagrams of Poros City and diagrams of Poros structure extracted by Taihu stone, and methodology differs in organisation methods. 


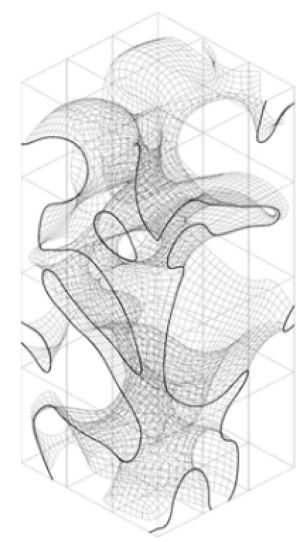

(a) Diagrams of Poros City
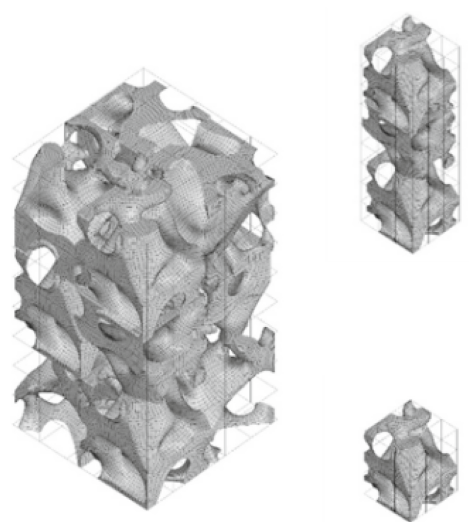

(b) Diagrams of Poros structure

Fig. 11. (a) Diagrams of Poros City (b) Diagrams of Poros structure

\section{References}

1. Diao, H.: Taihu Stone in China. Shanghai Science and Technology Press (2008)

2. Zhang, T., Rainer, P., Zhang, H.: POROsCITY: An Experimental Design Studio for the $12^{\text {th }}$ International Architectural Exhibition/La Biennale di Vennezia 2010.03-2010.08. Southeast University Press (2017)

3. Liu, D.: YU YUAN STONE (2008). http://www.sothebys.com/en/auctions/ecatalogue/2016/ origo-collection-hk0684/lot.516.html

4. Oktay, E., Akay, H.U., Merttopcuoglu, O.: Parallelised structural topology optimisation and CFD coupling for the design of aircraft wing structures. Comput. Fluids 49, 141-145 (2011). https://doi.org/10.1016/j.compfluid.2011.05.005

5. Campana, E.F., Peri, D., Tahara, Y., Stern, F.: Shape optimisation in ship hydrodynamics using computational fluid dynamics. Comput. Methods Appl. Mech. Eng. 196, 634-651 (2006). https://doi.org/10.1016/j.cma.2006.06.003

6. Bijan, M., Olivier, P.: Shape Optimisation in Fluid Mechanics. Ann. Rev. Fluid Mech. 36(1), 255-279 (2004). https://doi.org/10.1146/ANNUREV.FLUID.36.050802.121926

7. Zuo, Z.H., Xie, Y.M., Huang, X.: Combining genetic algorithms with BESO for topology optimisation. Struct. Multidisc. Optim. 38(5), 511-523 (2009). https://doi.org/10.1007/s00 158-008-0297-5

8. Huang, X., Xie, Y.M.: A new look at ESO and BESO optimization methods. Struct. Multidisc. Optim. 35(1), 89-92 (2007). https://doi.org/10.1007/s00158-007-0140-4

9. Zhou, Y.Y., Liu, X.D., Hua, Z.L., Hu, G.Y., Gu, L., Chen, L.Q.: Study of the temporal and spatial differences of hydrodynamic characteristics of wind-induced current in Taihu Lake. Environ. Protect. Sci. 41(1), 51-56 (2015). https://doi.org/10.16803/j.cnki.ISSN.1004-6216. 2015.01.012

10. Liang, R.J., Zhong, J.H.: A three-dimensional numerical simulation of wind-driven water current in Taihu Lake. J. Lake Sci. 6(4), 289-297 (1994). https://doi.org/10.18307/1994.0401

11. Wang, J.W., Li, Y.P., Luo, L.C., Dai, S.J.: Field observation of vertical shear of wind-driven current in Taihu Lake. Water Resource Protect. 32(6), 98-116 (2016). https://doi.org/10.3880/ j.issn.1004-6933.2016.06.016 
12. Xie, Y.M., Zuo, Z.H., Lv, J.C.: Application of bi-directional evolutionary structural optimization on architectural design. Time+Architecure 5, 20-25 (2014). https://doi.org/10.13717/j. cnki.ta.2014.05.004

13. Xu, Q.: The manifestation and cultural connotation of artistic symbols in Taihu stone. Art Apprec. 30, 32-33 (2020). CNKI:SUN:YSPJ.0.2020-30-021

14. Huang, X., Xie, Y.M., Burry, M.C.: Advantages of Bi-Directional Evolutionary Structural Optimization (BESO) over Evolutionary Structural Optimization (ESO). Adv. Struct. Eng. 10(6), 727-737 (2007). https://doi.org/10.1260/136943307783571436

15. Yin, T.T.: Application of element of Suzhou Taihu stone in modern architecture and landscape design. Beauty Times (urban version) 09, 21-22 (2020). CNKI:SUN:MEIC.0.2020-09-011

16. Xie, Y.M., Steven, G.P.: A simple evolutionary procedure for structural optimization. Comput. Struct. 49(5), 885-896 (1993). https://doi.org/10.1016/0045-7949(93)90035-C

17. Xie, Y.M., Steven, G.P.: Optimal design of multiple load case structures using an evolutionary procedure. Eng. Comput. 11(4), 295-302 (1994). https://doi.org/10.1108/026444094107 99290

18. Liang, X., Wang, Y.: The advancement and practice of the idea of architectural phenomenon of Steven Holl's. World Arch. 04, 114-117 (2012). https://doi.org/10.16414/j.wa.2012.04.027

Open Access This chapter is licensed under the terms of the Creative Commons Attribution 4.0 International License (http://creativecommons.org/licenses/by/4.0/), which permits use, sharing, adaptation, distribution and reproduction in any medium or format, as long as you give appropriate credit to the original author(s) and the source, provide a link to the Creative Commons license and indicate if changes were made.

The images or other third party material in this chapter are included in the chapter's Creative Commons license, unless indicated otherwise in a credit line to the material. If material is not included in the chapter's Creative Commons license and your intended use is not permitted by statutory regulation or exceeds the permitted use, you will need to obtain permission directly from the copyright holder.

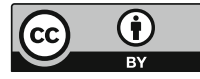

